\title{
La cultura tributaria como el raciocinio de los evasores contributivos
}

\author{
The tax culture as the reasoning of the levy evaders \\ A cultura fiscal como o raciocínio das \\ pessoas que evadem impostos \\ Máster Emmanuel Guzmán Rodríguez
}

\section{RESUMEN}

La investigación identifica y explica las razones de la sociedad para evadir sus responsabilidades contributivas. Este raciocinio es la composición de la moral social, normas sociales y la ética social. Estos tres factores tienen la capacidad de influenciar la cultura tributaria de cada país. Se discute que los diversos métodos y estrategias de evadir las responsabilidades contributivas son utilizados por una cultura de evasores.

Palabras clave: raciocinio contributivo, evasión contributiva, moral social, ética social, normas sociales, cultura tributaria.

\section{ABSTRACT}

The research identifies and explains the society reasons to evade their tax responsibilities. This reasoning is composed by the social moral, social norms and social ethics. These three factors have the capacity of influencing the tributary culture of each country. The study discusses different methods and strategies of evading the tax responsibilities by a culture of evaders.

Keywords: tax reasoning, levy evasion, social moral, social ethics, social norms, tributary culture.

\section{RESUMO}

A pesquisa identifica e explica as razóes da sociedade para evadir suas responsabilidades fiscais. Este raciocínio é a composição da Moral Social, normas sociais e a ética social. Estes três fatores têm a capacidade de influenciar a cultura tributária de cada país. Se discute que os diversos métodos e estratégias de evasão das responsabilidades contributivas são utilizados por uma cultura deevasão.

Palavras-chave: raciocínio tributário, evasão contributiva, moral social, ética social,normas sociais, cultura fiscal.

* Ciudadano Americano, Maestría en Contabilidad, Maestría en Contribuciones, Bachillerato en Contabilidad Pública, Sistema Ana G. Méndez, Universidad del Turabo, Puerto Rico. Correspondencia con el autor: emanguz@hotmail.com. 


\section{Introducción}

Los gobiernos del planeta Tierra comparten el problema de la evasión contributiva. Dilema que afecta a países como África y a China ya que los impuestos de los productos importados tienen una relación positiva, directa y elástica con las tasas tributarias (Van \& Channing, 2009). El gobierno de los Estados Unidos ha recolectado \$345 billones por debajo de lo estimado en los impuestos sobre los ingresos a dueños de negocios propios (Cleary, Karlinsky \& Bankman, 2009). Se ha considerado la ética social como la causa de las evasiones contributivas en cada país (Fagbemi et al., 2010). La evasión tributaria causa la disminución del ahorro, y reduce el crecimiento económico (Fagbemi et al., 2010). Este comportamiento distorsiona el comercio, y agita la infraestructura del Estado (Fattah, 2010). Alteran los objetivos del sistema gubernamental al recolectar cantidades inferiores a lo debido, y traen repercusiones socio-económicas como el ejemplo de la guerra civil en los Estados Unidos (Fagbemi et al., 2010). Hostilidad cívica comenzada por incongruencias en las normas y sus creencias sociales. Incluso estas diferencias estaban basadas en el derecho social de esclavizar a las personas para generar e incrementar su capital personal y familiar (Weinstein, 1984).

Luego de la guerra civil de los Estados Unidos de América (1865), la imposición de los impuestos ha creado una subcultura económica, en donde el único principio de las personas es de maximizar sus ingresos. La opinión general de las personas es indagar en formas de incrementar sus ganancias y retener las mismas (Limerick, 1987). La imposición de los gravámenes por el gobierno va en contra de esta ideología e investigan métodos de cómo lograr la evasión tributaria.

El autor Levi (2010) creó un modelo de la evasión tributaria en un esquema titulado "Cumplimientos de las Políticas Contributivas". En este modelo se explica el discernimiento de los contribuyentes en tres perspectivas: el tipo de negocio, el auditor fiscal y el contribuyente. Las tres perspectivas comienzan con la apariencia política relacionada con el deber social de cumplir con la política fiscal y con el sistema tributario. Las personas con negocios propios están predeterminadas a evadir los impuestos, porque realizan sus propias responsabilidades contributivas (Levi, 2010). El contribuyente puede facilitar o impedir la función del auditor si su relación es de servicio al cliente o de policía y ladrón. Es en esta etapa en que la autoridad del agente fiscalizador puede ser sobornada o el contribuyente puede completar una interferencia astuta. En la perspectiva del contribuyente se analiza la recompensa de evadir en contra de las probabilidades de ser auditado. Los evasores realizan un análisis sicológico compuesto de la secuencia de auditorías, sus técnicas de indagación, predeterminar el ánimo a utilizar con el auditor y la estructura del comercio. 
La psicología social representa el entendimiento de los gravámenes, las actitudes, creencias, normas sociales/personales, la oportunidad de evadir y la evaluación del contribuyente sobre su gobierno.

\section{Importancia del estudio}

El problema de la evasión contributiva es común en todos los gobiernos de nuestro planeta Tierra. Por ser un problema común no es irrelevante, es un obstáculo mundial que debilita la estructura económica de los gobiernos. Esta investigación busca brindar entendimiento de este dilema al investigar la raíz del razonamiento social para evadir o pagar los impuestos de su gobierno. A pesar de que el tema de la evasión contributiva es muy estudiado, no ha podido ser erradicado. Esta investigación brinda luz para nuevas investigaciones con el fin de eliminar este problema en común que afecta a todos los gobiernos del planeta Tierra.

\section{Metodología}

Este estudio es un trabajo derivado del estudio de Guzmán (2012). La metodología utilizada comienza al exponer el problema de la evasión contributiva. Luego de una extensiva revisión de literatura se delimitó el estudio a investigar el raciocinio de las personas para evadir el pago de sus responsabilidades contributivas. Esta delimitación es reflejada en las tres preguntas de investigación, que son contestadas con artículos empíricos y cualitativos revisados por pares y publicados en distintas revistas académicas. Por consecuencia, este estudio está basado en una revisión de artículos sobre la cultura tributaria, la norma social, la ética social y la moral social.

Este trabajo se considera un artículo cualitativo porque el diseño investigativo es de reporte, descriptivo y causal-explicativo que intenta definir, describir y explicar los factores que tienen influencia en la conciencia de los contribuyentes evasores. Estos constructos han sido identificados como la Norma Social, Ética Social y la Moral Social que forman parte de la variable dependiente identificada como la Cultura Tributaria de las personas.

\section{Preguntas de investigación}

Las preguntas tienen el objetivo de identificar el raciocinio que tienen los contribuyentes al evadir los gravámenes junto con los métodos y estrategias utilizadas para completar su evasión contributiva.

- ¿Cuáles son los cimientos de la evasión contributiva para los contribuyentes? 
- ¿Cómo las normas sociales, la ética social y la moral social influyen en la evasión de los gravámenes?

- ¿Cuáles son los métodos y estrategias utilizadas para la evasión contributiva?

\section{Los cimientos de la evasión contributiva}

Los cimientos de la evasión contributiva están en las razones de los contribuyentes. Raciocinios relacionados con la estructura de los beneficios sociales del gobierno hacia su población (Fagbemi et al., 2010). Si los beneficios públicos en la educación, seguridad y el sistema de salud están maltratados, son incompetentes y no buscan mejorarlos, proveen bases para una actitud evasiva (Fagbemi et al., 2010). Según Pashev (2005), el gobierno necesita demostrar responsabilidad y transparencia para no incrementar las cantidades de evasores al afectar la confianza de los contribuyentes hacia el sistema tributario (Fagbemi et al., 2010:5). En el estudio de Cleary et al. (2009) proponen la existencia de 14 factores que influyen en el raciocinio de las personas. En estos se incluyen: la edad, el género, la educación, el nivel de ingresos, la fuente de ingresos, influencia de compañeros, la ética, la equidad, la complejidad y las tasas contributivas. Los factores fuentes de ingresos y la influencia de compañeros son los más relevantes (Cleary et al., 2009).

En un estudio empírico para determinar las razones de las personas en participar en la evasión contributiva, se clasificaron las razones en 18 argumentos, razones descritas como la ética del contribuyente (McGee, López \& Yepes, s. f.). En la tabulación, los argumentos más sólidos -en estar de acuerdo en cometer evasión- resultaron ser la corrupción de los empleados del gobierno, discriminación por religión, raza o grupo étnico, que el gobierno desperdicie los fondos recaudados, encarcelación por puntos de vista políticos y la evasión por los judíos en Alemania ante el poder de Adolfo Hitler (McGee et al., s. f.). Otros planteamientos populares pero menos sólidos fueron: injusticia del sistema, capacidad de pagar, que los recaudos se utilicen en proyectos inmorales y que el gobierno esté en una guerra injusta (McGee et al., s. f.). Los argumentos más débiles fueron que todo el mundo evade las contribuciones y se evaden los impuestos porque la probabilidad de ser auditado es poca (McGee et al., s. f.). La ética social es una evaluación de las personas sobre las conductas y actitudes de su gobierno.

La evasión contributiva es causada por la cultura social, conocida como la cultura tributaria. La pobreza de las personas está relacionada con la economía informal (Danopoulos $\&$ Znidaric, 2007). Aunque la economía informal no significa evasión contributiva, las personas pueden llegar a no reportar sus ingresos por restricciones de los ingresos en las políticas del Estado (Danopoulos \& Znidaric, 2007). Las personas desempleadas, ancianos, granjeros, pensionados, y los segmentos vulnerables tienen una correlación fuerte entre la evasión contributiva y la economía informal (Danopoulos \& Znidaric, 2007). 
Esta fuerte relación existe por las siguientes circunstancias que forman parte de las características de vida de los evasores: la educación, el nivel social del ciudadano, percepción de una economía justa, el peso del nivel contributivo, los métodos y la percepción social de cómo el gobierno utiliza los recursos, la efectividad del sistema contributivo, el nivel de competencia por las agencias fiscalizadoras, la documentación de las actividades del gobierno, la naturaleza de los negocios y el tamaño del mercado, y la estructura económica del gobierno (Danopoulos \& Znidaric, 2007). Si la cultura tributaria es una de evasores, puede crear varios efectos negativos en la sociedad; los cuales incluyen la distribución de un peso contributivo injusto y no equitativo, reduce los ingresos del gobierno, interrumpe en el proceso de completar las políticas económicas al minimizar la efectividad de los métodos para colectar los impuestos, rompe las reglas de un mercado libre y competitivo, incrementa los impuestos regresivos, e influye a la sociedad negativamente sobre cumplir con las leyes del Estado (Danopoulos \& Znidaric, 2007).

En el estudio de Valero et al. (s. f.) confirman lo mencionado por Danopoulos \& Znidaric (2007) en su estudio cualitativo, en que la cultura de Venezuela tiene una actitud negativa y antiética hacia el gobierno. Actitud negativa que proviene por falta de un proceso educativo para crear conciencia, responsabilidad, y solidaridad en los contribuyentes (Valero et al., s. f.). La actitud o cultura tributaria puede ser modificada o creada por medio de políticas de control en las contribuciones, transparencia en los recaudos utilizados, planteamientos para cambiar el sistema contributivo, y la capacitación de los agentes fiscalizadores (Valero et al., s. f.:2). La falta de transparencia y responsabilidad en los recursos gubernamentales crea desconfianza para el sistema contributivo y para el gobierno (Fagbemi et al., 2010).

\section{La influencia de las normas sociales, la ética social y la moral Social en la evasión de los gravámenes}

Las ideologías de cada persona surgen del razonamiento. Graham (1999:2) menciona que las decisiones de las personas están basadas en su tradición, compuesta por: "sentido común familiar, o por su lealtad”. Las normas se aprenden de amigos, y si estos evaden las contribuciones es porque el riesgo de ser auditados es improbable (Cleary et al., 2009). Los dueños de negocios justifican su evasión, porque el gobierno les impide progresar (Cleary et al., 2009). Los dueños de negocios evaden las contribuciones por sus normas y las oportunidades obtenidas, y no por la moral, por la complejidad, ni por las políticas del gobierno (Cleary et al., 2009:29). Las normas sociales son influenciadas por el grupo ambiente, el preparador de planillas del contribuyente, y no por el gobierno (Cleary et al., 2009). Argumento que debe ser estudiado para hallar la influencia de la actitud errónea del gobierno en su sociedad. 
El estudio de Cleary et al. (2009) complementa la investigación de Fagbemi et al. (2010), porque mencionan que las razones de la evasión contributiva está en la ética del contribuyente. Ética con efectos de distorsionar el mercado perfecto y agitar la infraestructura del estado por medio de los fondos recaudados del gobierno (Fagbemi et al., 2010). En el estudio empírico realizado por Fagbemi et al. (2010:6) aluden en su primera hipótesis: "La evasión contributiva es ética algunas veces". La evasión de gravámenes es ética si el gobierno: discrimina a las personas por razón de religión o grupo étnico, empleados corruptos, trato injusto, incapacidad de pagar los impuestos, sistema contributivo desfavorable, los impuestos son muy altos, si la persona es extranjera, si el dinero recolectado es mal gastado, si es utilizado en proyectos no beneficiosos para el contribuyente, si el grupo ambiente evade los impuestos, si existe poca probabilidad de ser auditado, si los recaudos son utilizados para propósitos inmorales, si los recaudos son utilizados para propósitos injustos, si los impuestos no son tan altos, si las personas pueden pagar menos contribuciones, si el gobierno encarcela a las personas por su afiliación política, si el dinero recolectado es utilizado en proyectos no útiles, y si el dinero es utilizado en proyectos no beneficiosos (Fagbemi et al., 2010:9).

Sin embargo, Fagbemi et al. (2010:6) en su segunda hipótesis: "La evasión contributiva es más aceptable cuando el gobierno aparenta ser corrupto”. Resultó que la evasión es aceptable sólo si el gobierno: discrimina por razón de religión o grupo étnico, las personas son encarceladas por su afiliación política, y si las personas no tienen la capacidad de pagar sus impuestos. Los argumentos con más probabilidades de evasión son: discriminación por religión o grupo étnico, empleados corruptos, trato injusto, encarcelación por afiliación política, incapacidad de pagar los impuestos, tasas contributivas muy altas, sistema contributivo injusto, y si el contribuyente es extranjero (Fagbemi et al., 2010). Los argumentos más sólidos para cometer evasión son: la corrupción del gobierno, discriminación, capacidad de pagar los impuestos, y trato injusto (Fagbime et al., 2010). La ética corrupta ocurre cuando el agente auditor acepta dinero por parte de un evasor adinerado (Fagbemi et al., 2010:5). Según Acconia (2003) el nivel de corrupción depende de los factores: evasor con abundancia de dinero y el salario del agente auditor (Fagbemi et al., 2010:5).

La ética social se basa en tres tipos de opiniones, primero la opinión no ética son las personas que cumplen con sus responsabilidades contributivas (Fagbemi et al., 2010). Deberes contributivos que los autores Cohn (1998) y Smith \& Kimball (1998) dicen que existen por tres razones: la creencia de cada individuo en pagar los impuestos que el gobierno establece; porque los individuos tienen una responsabilidad con los otros miembros de la comunidad, y las creencias de su religión (Fagbemi et al., 2010:5). Segundo, la opinión anarquista, se puede utilizar para identificar las actitudes de las personas hacia el gobierno -moral social (Fagbemi et al., 2010:6)-. Esta 
opinión puede ser descrita como personas con una moral en contra del gobierno que entorpece el sistema tributario. Y tercero, la opinión circunstancial, las personas tienden a justificar su evasión o condenarla (Fagbemi et al., 2010:6). Justificación o condenación basadas en su moral, inclinación religiosa, y creencias (Fagbemi et al., 2010:6). La cultura tributaria desde la conciencia de las personas hacia el sistema tributario, la moral social desde las perspectivas del ciudadano hacia su gobierno en la discriminación, corrupción, probabilidad de auditorías, y la malversación de fondos (Cleary et al., 2009). La ética social es utilizada para evaluar al gobierno y justificar la evasión contributiva.

\section{Los métodos y estrategias utilizadas para la evasión contributiva}

Los evasores ven las auditorías como un juego de probabilidades y ellos siempre juegan en contra del gobierno. Su juego de evadir las contribuciones se manifiesta en dos formas: reportan los precios de lo importado por debajo del valor, y/o reclasifican la categoría de la mercancía en tasas inferiores (Van \& Channing, 2009). Las formas de evadir los impuestos ocurren al no reportar todos los ingresos, reclamar deducciones, y/o créditos falsos (Soyode y Kajola, 2006). Mientras que Kay (1980) opina que la evasión ocurre al manipular la información. La evasión ocurre al malinterpretar o esconder la información (Fagbemi et al., 2010). Y Cleary et al. (2009) expresan que la evasión acaece cuando los contribuyentes reportan cantidades menores a los ingresos realmente recibidos en las transacciones de efectivo.

Otras formas de evadir las contribuciones ocurren en los empleos informales como: la jardinería, lavado de automóviles, mecánicos a domicilio, remolque, y entre otros trabajos esporádicos que no reportan ni pagan sus gravámenes (Cleary et al., 2009). Los dueńos de pequeños negocios tienden a no reportar parte o el total del efectivo recibido; sin embargo, reportan todos los ingresos recibidos con cheques, tarjetas de crédito, tarjetas de débito, y giros (Cleary et al., 2009). Los reportan porque estos métodos de pagos pueden ser rastreados (Cleary et al., 2009). Los dueños de negocio utilizan métodos como reportar el $85 \%$ de las ventas, y no reportan entre el 10\% al $20 \%$ de las ventas, pasan los cheques recibidos de mano en mano porque estos eran tan buenos como el efectivo, cambian los cheques por efectivo, ofrecen un descuento de $20 \%$ si pagan en efectivo, y otros nunca depositan el dinero recibido en efectivo en las entidades financieras (Cleary et al., 2009). Un dueño de negocio mencionó: "si vas a evadir hazlo en las deducciones o en el ingreso reportado, pero nunca en ambas" (Cleary et al., 2009:16). Dueños de negocios mencionan que todo el dinero recibido en efectivo debe ser gastado en objetos personales que preserven su valor, guardarlo dentro de cajas fuertes en sus casas, o invertirlo en el negocio (Cleary et al., 2009). 
La evasión tributaria puede ocurrir en cualquier entidad como corporaciones, sociedad, dueños propios y entidades conductos (Cleary et al., 2009). En la evasión contributiva colectiva participan bancos, empresas, personas naturales del mismo país y de países extranjeros (Ralston, 2010). Los evasores en grupos tienen un criterio para perpetrar sus actividades: buscan países extranjeros con tasas convenientes, crean un fideicomiso y una empresa dentro del gobierno donde quieren hacer negocios; y luego todo el dinero obtenido se envía al fideicomiso para transferirlo a una corporación extranjera (Ralston, 2010). En sus actividades pueden repartir ganancias y pagos de servicios ficticios sin pagar contribuciones en ambos gobiernos. Este tipo de evasión tiene tres elementos en común una empresa, un comercio interestatal o internacional, y un patrón de sobornos (Ralston, 2010).

Algunas de las estrategias escabullidas incluyen: minimizar los ingresos, ganancias, volumen de ventas, o incrementar los gastos deducibles, reducir la escala contributiva asignada, no reportar a los trabajadores, transferir las ganancias a otros países con tasas contributivas preferenciales, manipulación de precios entre compañías relacionadas y sus subsidiarias, incrementar los precios de lo importado, y reducir los precios de lo exportado (Dumitrescu, 2010). La estrategia es completada al mantener recibos ficticios de todos los gastos operacionales para cubrir sus actos ilícitos (Dumitrescu, 2010).

\section{Discusión de los resultados}

La ética social es el cúmulo de acciones del Estado evaluadas por su población. Actividades evaluadas con la moral y normas de cada persona, que luego forman parte de la percepción aprendida del contribuyente que se resume en la cultura tributaria. Las normas sociales explican el porqué de evadir las contribuciones. Estas son la acumulación del raciocinio personal/social basada en los factores observados en el gobierno. Si las acciones del Estado son corruptas, la cultura tributaria también. Un auditor del gobierno con este razonamiento, será sobornado por un evasor adinerado. Como la ética social, las personas desarrollan una moral social/personal hacia el gobierno. La ética social toma en consideración el perdón contributivo a los evasores, las fiscalizaciones contributivas ineficientes -auditorías, penalidades, y cargos criminales-, el porciento de las tasas contributivas, corrupción observada y aprendida del gobierno, las injusticias sociales, el trato humano del personal de las organizaciones gubernamentales, y la estructura del sistema contributivo. Las personas aplican su propia ética en distintas situaciones, y la misma es creada con las acciones aprendidas, y criticadas con la moral personal/social de cada individuo. Cuando las personas tienen la responsabilidade de cumplir con los gravámenes contributivos, sus 
acciones toman en consideración las actitudes del gobierno la analizan con su ética social y deciden si cumplen con sus contribuciones.

Para el buen funcionamiento de cualquier enmienda o nuevo impuesto es necesario influenciar el pensamiento del contribuyente. En África sub-Sahariana se incrementaron los gravámenes de los artículos importados en $1 \%$ sin considerar la opinión pública y resultó en un incremento evasivo por 1,4\% (Van \& Channing, 2009). La evasión es basada en el raciocinio de las probabilidades de ser fiscalizados y el ahorro al cometerla (Fagbemi et al., 2010). A mayores tasas contributivas superior es la evasión tributaria (Fagbemi et al., 2010). Dilema con solución pero se considera que la ética social, la moral social, y las normas sociales influyen en los agentes fiscalizadores al ser sobornados por el evasor identificado (Fagbemi et al., 2010). Los agentes se afectan si comparten el conocimiento de que el Estado es corrupto, discriminante y utiliza mal sus fondos (McGee et al., s.f.).

El problema de la evasión contributiva es uno más profundo que sólo lo económico, financiero y legal (Valero et al.). Su respuesta fue profundizar en la relación Estado y ciudadano. Investigar en otros conceptos y categorías conexos a la vida social, política, y cultural para la elaboración de las políticas tributarias. En estos conceptos y categorías se incluyen: la ciudadanía, deberes y derechos del contribuyente, servicios públicos, justicia social, valores culturales, vida pública, vida ciudadana, legalidad, legitimidad, prácticas y representaciones sociales (Valero et al.). Estos conceptos y categorías se resumen dentro de la ética y la cultura tributaria (Valero et al.). La cultura tributaria puede ser modificada o creada con la conciencia del contribuyente (Valero et al.). Este entendimiento se crea con ayuda de la educación, la solidaridad, la historia económica del Estado, la idiosincrasia del pueblo, y la necesidad de transparencia en el uso del dinero público (Valero et al.).

Figura 1.0. La conciencia del contribuyente evasor como resultado de la cultura tributaria.

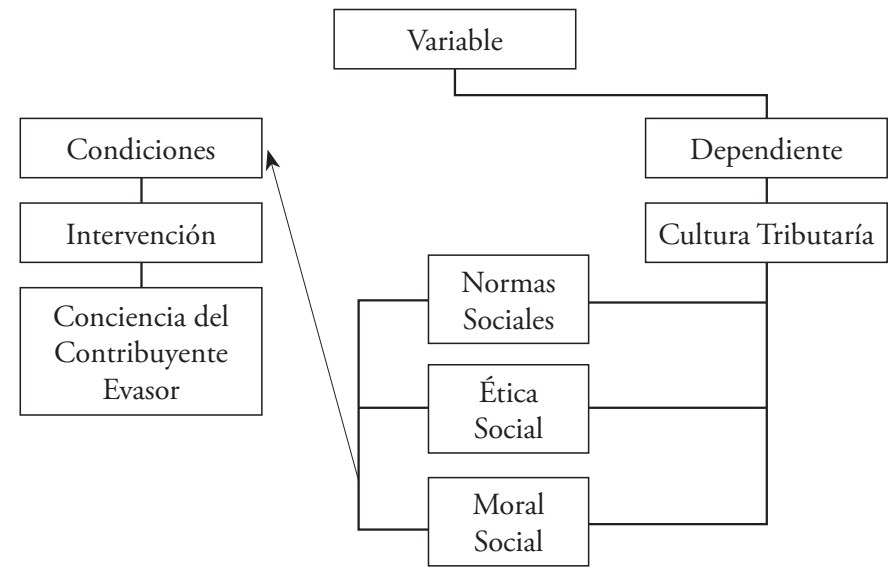

Fuente: esquema derivado del estudio de Guzmán (2012). 
A diferencia del estudio de Guzmán (2012), este esquema considera la cultura tributaria de una sociedad o grupo de personas como dependiente. Depende de los factores de normas sociales, la ética social y la moral social para crear una conciencia contributiva que puede resultar en la de evasor contributivo. La semejanza de esta ilustración con la de Guzmán (2012), es que ambas reconocen que la conciencia del contribuyente puede ser creada y modificada con una intervención de distintas condiciones que influyan en las normas sociales, en la ética social y la moral social de una sociedad en específico. Como indican Gioia \& Pitre (1990), las realidades del presente eran perspectivas del pasado. Estas realidades pueden cambiar o ser modificadas con nuevas perspectivas. Es necesaria la intervención del gobierno para influenciar la conciencia de sus contribuyentes.

\section{Conclusión}

En conclusión la raíz de la evasión contributiva reside en las actitudes del gobierno y del sistema tributario hacia su sociedad. Estas actitudes tienen una relación directa entre el gobierno y la evasión de los gravámenes. Se pudo identificar que las razones de la sociedad para justificar la evasión contributiva pueden ser utilizadas para determinar la existencia de la evasión de gravámenes en distintos sectores sociales y si el gobierno está susceptible a una cultura tributaria de evasores contributivos.

La creación de una cultura de contribuyentes honestos depende de la situación económica del país, las actitudes del gobierno, y la cultura tributaria. El gobierno de cada país necesita crear estrategias políticas, económicas, legales y educativas para influenciar los factores de la cultura contributiva. Las normas sociales explican el porqué de evadir las contribuciones, la ética social son las justificaciones, y la moral social es influenciada por el ambiente, amigos, y la familia del contribuyente. Es necesaria la creación de un cuestionario con los hallazgos discutidos de Cleary et al. (2009), Danopoulos \& Znidaric (2007), Valero et al. (s.f.), Dumitrescu (2010) y Fagbemi et al. (2010), para poder identificar el porqué, cómo, dónde y quiénes tienen una cultura contributiva de evasores.

El gobierno necesita involucrarse y resolver los problemas de su sociedad, e incluso tomar medidas para influenciar la percepción social de su rol en el país. El sistema contributivo tiene la función de multiplicar el flujo de efectivo del gobierno; sin embargo, su eficiencia depende de las actitudes de su población. La cultura tributaria es un fenómeno social/económico que afecta al gobierno y sus servicios públicos.

La evasión contributiva es un problema de todos los gobierno del planeta Tierra que causa una disminución en el flujo de efectivo, el cual puede afectar el total de beneficios 
otorgados a su población, e incluso puede causar el incumplimiento del pago de las deudas de un gobierno por escasez de flujo de efectivo. Problema que puede disminuirse al implementar nuevos impuestos; sin embargo, esto puede influenciar negativamente en la cultura tributaria.

\section{Bibliografía}

Agosto Reyes, Alwin (1990). Percepción de los contribuyentes con relación a la Reforma Contributiva comparada con la Antigua Ley de Contribuciones Sobre Ingresos (Master Thesis). Disponible en la Biblioteca Virtual de la Universidad del Turabo.

Bersoff, David M. (1999). "Explaining unethical behavior among people motivated to act prosocially". Journal of Moral, Vol. 28, No. 4/ Education.

Carlsen, Melody A. (1992). "Group health benefits fraud: who's winning a lose-lose game?” [Peer commentary on various papers]. Obtenido en los Jornales Académicos de la Biblioteca Virtual de la Universidad del Turabo.

Cleary, Morse Susan; Stewart, Karlinsky \& Joseph, Bankman (2009). "Cash businesses and tax evasion”. Stanford Law \& Policy Review, Vol. 20:1.

Código de Rentas Internas (2011). Código de Rentas Internas para un Nuevo Puerto Rico, Ley Núm. 1 de 31 de enero de 2011. Obtenido el 3 de marzo de 2011, en http://www.hacienda.gobierno.pr/\#

Cooper, D. R. \& Schindler, P. S. (2008). Business research methods (10 ${ }^{\mathrm{ma}}$ Ed.). Singapore, McGraw Hill: pág. 6, 19, 142-144, 162-167 \& 308.

Crumbley, Larry D.; Friedman, Jack P. \& Anders, Susan B. (1994). Dictionary of tax terms. Barron's Educational Series: Hauppauge, New York 11788.

Danopoulos, Constantine P. \& Boris, Znidaric (2007). "Informal economy, tax evasion, and poverty in a democratic setting: Greece". Mediterranean Quarterly 18:2. doi: 10.1215/10474552-2007-006.

Demidova, Olga \& Im, Gym Dya (s. f.). "How people of different countries consider an infringement of the law: the comparative econometric analysis". Contemporary Legal \& Economic Issues.

Downing, Douglas (2009). Dictionary of mathematics terms (3 ${ }^{\text {th }}$ Ed.). New York, Barron's: Hauppauge.

Dumitrescu, Constantin Dan (2010). "Tax fraud-specific forms of manifestation in the underground economy". Annals of DAAAM for 2010 \& Proceedings of the $21^{\text {st }}$ International DAAM Symposium, Volume 21, No. 1, ISSN 1726-9679. ISBN 978-3-901509-73-5. 
Dzhumashev, Ratbek \& Emin, Gahramanov (2010). "A growth model with income tax evasion: some implications for Australia". The Economic Record, Vol. 86, No. 275, pp. 620-636.

Fagbemi, Temitope Olamide; Olayinka, Marte Uadiale \& Abdurafiu, Olaiya Noah (2010). "The ethics of tax evasion: perceptual evidence from Nigeria". European Journal of Social Sciences, Volume 17, Number 3 (2010).

Fattah, Chaka (2010). "Déjà vu all over again: reexamining fundamental tax reform and evaluating the feasibility of a transaction tax in the $111^{\text {th }}$ congress". Harvard Law School Journals, Vol. 47.

Gifis, Steven H. (2010). Law dictionary (6th ed.). Barron's Educational Series: Hauppauge, New York 11788.

Gioia, Dennis A. \& Pitre, Evelyn (1990). "Multiparadigm perspectives on theory building". The Academy of Management Review, Vol. 15, No. 4 (Oct., 1990). pp. 584-602.

Giroux, Hary (s. f.). "Great events in accounting \& business history". MAYS Business School at Texas A\&M University. Obtenida en: http://acct.tamu.edu/giroux/timeline.html.

Graham, Harrison (1999). "Corruption, development theory and the boundaries of social change". Contemporary Politics, Volume 5, Number 3.

Guzmán Rodríguez, Emmanuel (2012). "Estudio Cualitativo sobre la Influencia Gubernamental en la Ética Social, Normas Sociales, La Moral Social y La Cultura Tributaria de los Contribuyentes en la Evasión Contributiva”. [Monografía]. Accesible en la Biblioteca Virtual de la Universidad del Turabo.

Hao, Yufan (1999). "From Rule of Man to Rule of Law: an unintended consequence of corruption in China in the 1990s". Journal of Contemporary China, 8(22), 405-423.

Johnston, Robert D. (1998). "Beyond 'the west': regionalism, liberalism, and the evasion of politics in the New Western History". Rethinking History, 2:2, pp. 239-277.

Kirchler, Erich; Muehlbacher, Stephan; Hoelzl, Erik \& Webley, Paul (2009). "Effort and aspirations in tax evasion: experimental evidence". Applied Psychology: An International Review, 2009, 58 (3), 488-507. doi: 10.1111/j.14640597.2009.00403.x.

Levi, Michael (2010). "Serious tax fraud and noncompliance: a review of evidence on the differential impact of criminal and noncriminal proceedings". American Society of Criminology. Volume 9, Issue 3/Criminology \& Public Policy. 
Lima, F. W. S. \& Georg, Zaklan (2008). Multi-agent-based approach to tax morale. International Journal of Modern Physics C. Vol. 19, No. 12 (2008) 1797-1808.

McCabe Coyle, Barbara \& Stream, Christopher (2006). "The chicken or the egg: a recent history of public opinion and tax reform in Florida". Journal of Public Budgeting, Accounting \& Financial Management, 18 (2), 167-191, Summer 2006.

McGee, Robert W.; Ho, Simon S., M. \& Li Annie Y., S. (2007). "A comparative study on perceived ethics of tax evasion: Hong Kong vs the United States". Journal of Business Ethics (2008). 77:147-158, doi: 10.1007/s10551-006-9304-1.

McGee, Robert W.; López Paláu, Silvia \& Yepes López, Gustavo A. (s. f.). “Es ética la evasión de impuestos?: estudio empírico de opinión en Colombia”.

McKeever, Gráinne (1999). "Fighting fraud: An evaluation of the government's social security fraud strategy". Journal of Social Welfare and family Law, 21(4) 1999: 357-371.

Pitts, Robert E. \& Wittenbach, James L. (1981). "Tax Credits as a Means of Influencing Consumer Behavior". Journal of Consumer Research, Vol. 8, December 1981.

Ralston, Chad P. (2010). "Going it alone: a pragmatic approach to combating foreigneffected tax evasion”. Emory International Law Review, Vol. 24.

Real Academia Española (2001). Diccionario de la lengua española (22da ed.). Real Academia Española: Madrid, España.

Rechberger, Silvia; Hartner, Martina; Kirchler, Erich \& Hämmerle, Katharina Franziska (2010). "Tax amnesties, justice perceptions and filing behavior: a simulation study”. Law \& Policy, Vol. 32, No. 2, April 2010. ISSN 0265-8240.

Rodríguez, Francisco (2004). "Inequality, redistribution and rent-seeking". Economics \& Politics, Volume 16, No. 3, 0954-1985, November 2004.

Sánchez, R. C. (2005). Redacción Comercial (2 ${ }^{\text {da }}$ Ed.). Universidad de Puerto Rico: San Juan Puerto Rico.

Tavares, José C. \& Iglesias, Mar (2010). "Dimensiones de las atribuciones causales de evasión de impuestos en Portugal”. Revista de Psicología del Trabajo y de las Organizaciones, Vol. 26, n. 1,-págs. 73-78. doi: 10.5093/tr2010v26n1a5.

Tax Fraud-Specific Forms of Manifestation in the Underground Economy (2010). Annals of DAAAM for 2010 \& Proceedings of the $21^{\text {st }}$ International DAAAM Symposium, Volume 21, No. 1, ISSN 1726-9679, ISBN 978-3-90150973-5, Editor B. Katalinic, Published by DAAAM International, Vienna, Australia, EU, 2010. 
Teisch, Jessica B. (s, f.). "From the dust bowl to California: the beautiful fraud". Obtenido el 1 de abril de 2012, en los Jornales Académicos de la Biblioteca Virtual de la Universidad del Turabo.

Terrel, Ellen (2004). History of US income tax. The Library of Congress: Researchers. Obtenida en http://www.loc.gov/rr/business/hottopic/irs_history.html

Thin, María (2008). "The trouble with tax havens: the need for new legislation in combating the use of offshore trusts in abusive tax shelters". Columbia Journal of Law and Social Problems, 41 no3 Spr. 2008.

Thomas, C. R. \& Maurice, C. S. (2008). Managerial economics: foundation of business analysis and strategy (10 ${ }^{\mathrm{ma}} \mathrm{Ed}$.). New York: McGraw Hill Irwin.

Torgler, Benno \& Schneider, Friedrich (2005). "Attitudes towards paying taxes in Austria: an empirical analysis". 32: 231-250/ Empírica, doi: 10.1007/ s10663-004-8328-y.

Torgler, Benno (2008). "What do we know about tax fraud? An overview of recent developments”. Social research, Vol. 75: No.4: Winter 20081239.

Valero, Mary Thais; Ramírez de Egáñez \& Moreno Briceño, Fidel (s. f.). "Ética y cultura tributaria en el contribuyente". Daena: International Journal of Good Conscience, 5(1) 58-73. ISSN 1870-557X.

Van Dunem, João Ernesto \& Channing, Arndt (2009). "Estimating border tax evasion in Mozambique". Journal of Development Studies, Vol. 45, No. 6, 10101025, July 2009.

Weinstein, Bernard L. (1984). "Energy, taxes and growth. The future of advanced societies". Obtenido el 1 de marzo de 2012, en los Jornales Académicos de la Biblioteca Virtual de la Universidad del Turabo.

\section{Cómo citar este artículo:}

Guzmán, Emmanuel (2014). "La cultura tributaria como el raciocinio de los evasores contributivos”, Oikos No 38, 23-36, Escuela de Administración y Economía, Universidad Católica Silva Henríquez (UCSH), Santiago de Chile. [http://ediciones.ucsh.cl/revistas.php]

Fecha de recepción: 26 / 07 / 2014

Fecha de aceptación: 26 / 12 / 2014 